\title{
Article \\ Local Drug Delivery to the Entire Cochlea without Breaching Its Boundaries
}

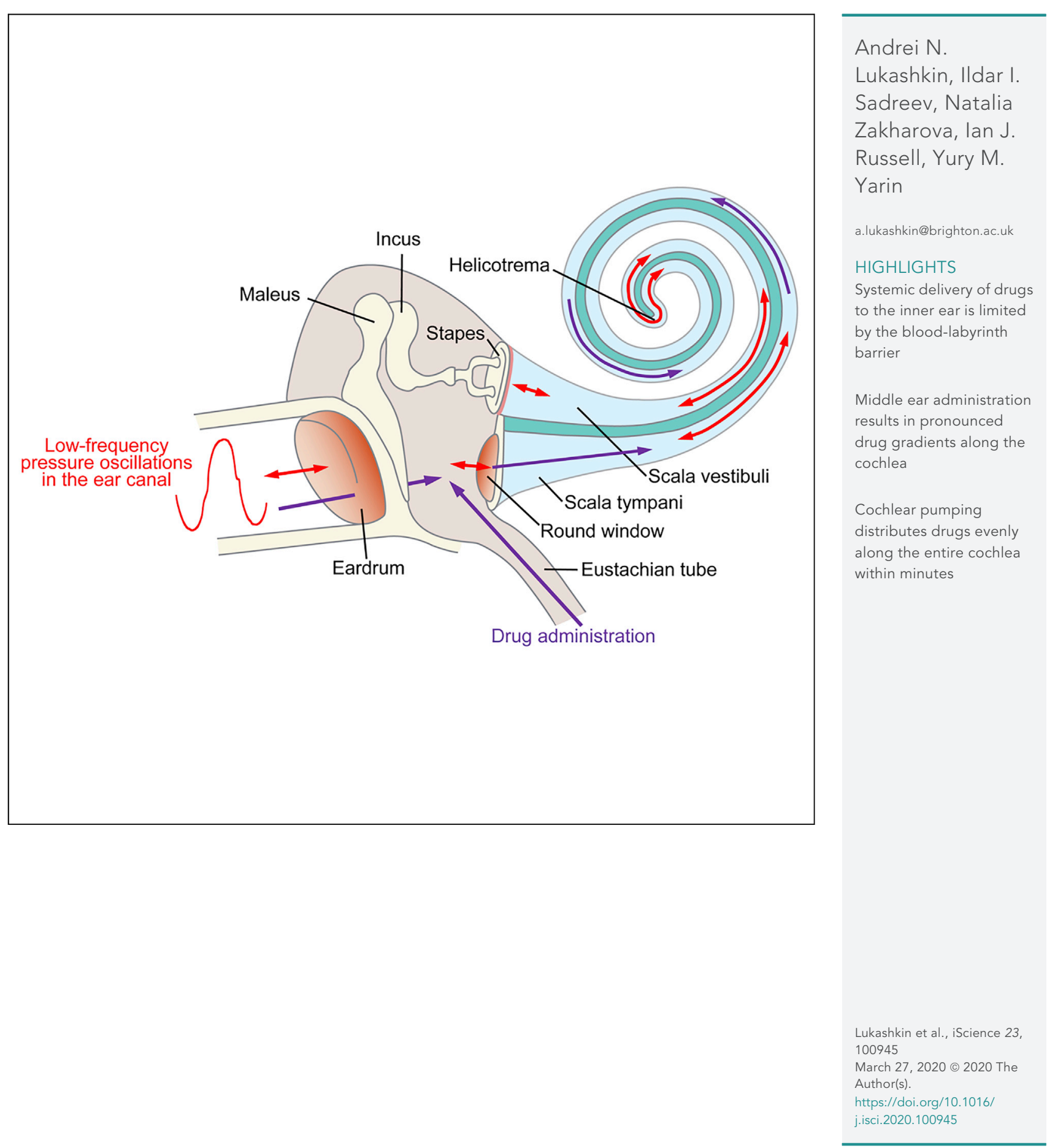




\title{
Article
}

\section{Local Drug Delivery to the Entire Cochlea without Breaching Its Boundaries}

\author{
Andrei N. Lukashkin, ${ }^{1,2,6, *}$ Ildar I. Sadreev, ${ }^{3}$ Natalia Zakharova, ${ }^{4}$ Ian J. Russell, ${ }^{1}$ and Yury M. Yarin ${ }^{5}$
}

\begin{abstract}
SUMMARY
The mammalian cochlea is one of the least accessible organs for drug delivery. Systemic administration of many drugs is severely limited by the blood-labyrinth barrier. Local intratympanic administration into the middle ear would be a preferable option in this case, and the only option for many newly emerging classes of drugs, but it leads to the formation of drug concentration gradients along the extensive, narrow cochlea. The gradients are orders of magnitude and well outside the therapeutic windows. Here we present an efficient, quick, and simple method of cochlear pumping, through large-amplitude, low-frequency reciprocal oscillations of the stapes and round window, which can consistently and uniformly deliver drugs along the entire length of the intact cochlea within minutes without disrupting the cochlear boundaries. The method should facilitate novel ways of approaching the treatment of inner ear disorders because it overcomes the challenge of delivering therapeutics along the entire cochlear length.
\end{abstract}

\section{INTRODUCTION}

Reliable, efficient, and uniform drug delivery to the cochlea remains an unsolved challenge and is a major barrier to the prevention or treatment of inner ear disorders. The mammalian cochlea is one of the least accessible organs for drug delivery (Salt and Plontke, 2009; Rivera et al., 2012; El Kechai et al., 2015; Hao and Li, 2019). Systemic administration of many drugs, notably the most frequently used corticosteroids and aminoglycoside antibiotics, is severely limited by the blood-labyrinth barrier (Salt and Hirose, 2018). Direct injection into the cochlea is limited by the requirement for surgery for access and does not guarantee uniform drug delivery along the cochlea.

Several potential therapeutic compounds to treat inner ear disorders are under clinical investigation. This comprises old and newly emerging classes of drugs and therapies including corticosteroids, local anaesthetics, antioxidants, apoptosis inhibitors, neurotransmitters and their antagonists, monoclonal antibodies, growth factors, signaling pathway regulators, and genetic material (see Devare et al., 2018; $\mathrm{HaO}$ and Li, 2019). A recent review identified 43 biotechnology companies currently pursuing experimental compounds for inner ear therapy (Schilder et al., 2019). All such efforts are, however, restricted by our inability to reliably deliver such compounds into the cochlea.

Intratympanic administration of drugs (Schuknecht, 1956) relies on their remaining in contact with the round window (RW) (a membranous opening in the bony wall of the cochlea into the middle ear) long enough to allow their diffusion into the perilymph of the scala tympani (ST). The ability of drugs to pass through the RW does not, however, guarantee their effective distribution along the cochlear spiral. Drug distribution in the ST is limited by the low flow rate of perilymph within the cochlea and by the cochlear geometry. The longitudinal flow of perilymph in the cochlea has been shown to be relatively slow, if present at all (Ohyama et al., 1988), and drug distribution in the perilymph is dominated by passive diffusion. Passive diffusion along the ST is, however, constrained because the cochlea is a relatively long and narrow tube with a cochlear cross-section that decreases gradually from the RW at the base to the apex.

Direct measurements of the distribution of marker ions and contrasting agents (Saijo and Kimura, 1984; Salt and Ma, 2001; Haghpanahi et al., 2013), corticosteroids (Hargunani et al., 2006; Plontke et al., 2008; Grewal et al., 2013; Creber et al., 2018), and antibiotics (Imamura and Adams, 2003; Mynatt et al., 2006; Plontke et al., 2007) or measurements of the physiological effects of drugs (Chen et al., 2005; Borkholder et al., 2010) have demonstrated that the concentration of substances applied to the RW is much higher in the cochlear base than in the apex.
${ }^{1}$ Sensory Neuroscience Research Group, School of Pharmacy and Biomolecular Sciences, University of Brighton, Brighton BN2 4GJ UK

${ }^{2}$ Centre for Regenerative Medicine and Devices, University of Brighton, Brighton BN2 4GJ, UK

${ }^{3}$ Faculty of Medicine, Department of Medicine, Imperial College, London SW7 2AZ, UK

${ }^{4}$ Otophysica, Uckfield TN22 2DN, UK

${ }^{5}$ ENT Practice, Dresden 01399, Germany

¿Lead Contact

*Correspondence: a.lukashkin@brighton.ac.uk https://doi.org/10.1016/j.isci. 2020.100945 
A large number of methods, including intracochlear administration, cochleostomy, and canalostomy, have been proposed for solving the problem of uniform drug distribution along the cochlea, but only two current strategies address this problem without breaching the boundaries of the intact cochlea (e.g., see El Kechai et al., 2015). The first strategy relies on retaining drugs in contact with the RW to allow drug diffusion into the cochlear apex. Notable examples of devices designed for this purpose include microwicks, osmotic pumps, etc. Hydrogel-based drug delivery systems also allow retention of therapeutics in the middle ear in contact with the RW. The problem with this strategy is that retention of drugs at the RW leads to their establishing steady-state concentration gradients along the cochlea, which depend on the relationship between diffusion and clearing (Salt and Ma, 2001; Sadreev et al., 2019), but the base-to-apex gradients can still be very pronounced.

The second strategy, although relatively non-invasive to the cochlea, requires development of more complex drug formulations. The technique employs drug-loaded nanoparticles, which could be used to take advantage of the anatomical and cellular features of the cochlea, which enable drug uptake through routes and pathways other than the ST route (Buckiová et al., 2012; Glueckert et al., 2018). Magnetically driven, drug-loaded magnetic nanoparticle can also be actively distributed along the entire cochlea (Ramaswamy et al., 2017).

Here we demonstrate that cochlear pumping (CP), through pressure oscillations in the ear canal at frequencies low enough to avoid damage to the cochlear sensory apparatus, can consistently and uniformly deliver drugs along the entire length of the intact cochlea within minutes without disrupting the cochlear boundaries.

\section{RESULTS}

\section{Cochlear Pumping at Low Frequencies Does Not Cause Elevation of Hearing Thresholds}

When inaudible low-frequency air pressure oscillations are presented at the ear canal, they are transmitted to the stapes, which causes back and forth cochlear fluid movements through the scala vestibuli (SV) and ST coupled via the helicotrema. The RW works as a pressure relief valve during these movements and moves in counter phase with the stapes because the cochlear bony wall and fluid are poorly compressible. The lowfrequency pressure changes within the cochlea are, however, shunted by the helicotrema and do not cause excitation of the traveling wave and stimulation of the sensory epithelium. In fact, shunting of lowfrequency perilymph flow, which occurs, for example, during the middle ear muscle reflex, thereby preventing cochlear overstimulation, is the previse role of the helicotrema (von Békésy, 1960). The high-frequency slope of the helicotrema mechanical filter is not, however, infinitely steep ( $6 \mathrm{~dB}$ /octave in guinea pigs and $12 \mathrm{~dB}$ /octave in humans; Marquardt et al., 2007) and the choice of CP frequency for drug delivery is critical to prevent cochlear damage. In our experiments, low-frequency air pressure oscillations applied to the ear canal at $4 \mathrm{~Hz}$, which caused large-amplitude ( $80 \mu \mathrm{m}$ peak-to-peak) stapes displacement (see Transparent Methods), did not elevate the threshold of the compound action potential (CAP) of the auditory nerve (Figure 1A).

\section{Cochlear Pumping Promotes Even Distribution of Drugs along the Cochlear Spiral}

The ability to distribute drugs uniformly along the entire cochlear spiral using relatively large, lowfrequency periodic displacement of fluid in the ST and SV was demonstrated in our experiments with application of salicylate to the RW. Salicylate readily passes through the RW (Borkholder et al., 2014; Sadreev et al., 2019). To monitor salicylate diffusion along an intact guinea pig cochlea in vivo, we utilized the suppressive effect of salicylate on cochlear amplification by blocking the outer hair cell somatic motility (Russell and Schauz, 1995; Hallworth, 1997). We measured the elevation of the CAP thresholds caused by salicylate at different frequencies, which, due to cochlear tonotopicity, corresponds to different distances from the RW (Greenwood, 1990).

Salicylate did not cause elevation of the CAP threshold responses for frequencies below $5 \mathrm{kHz}$, which corresponds to about $45 \%$ of the total cochlear length from the base, when it diffused through the cochlea passively (Sadreev et al., 2019). The calculated gradient of base-to-apex salicylate concentration was about 13 orders of magnitude. When, however, placement of salicylate solution on the RW was followed by CP, i.e., by 5 -min cycles of large-amplitude $(80 \mu \mathrm{m}$ peak-to-peak), low-frequency $(4 \mathrm{~Hz})$ stapes movements caused by pressure oscillations in the ear canal, the CAP threshold was elevated throughout the entire $1-30 \mathrm{kHz}$ frequency range tested (Figures $1 \mathrm{~B}, 2$, and 3). This corresponds to about $75 \%$ of the total cochlear length from the base (Greenwood, 1990). Partial recovery of the CAP thresholds after washing out salicylate 


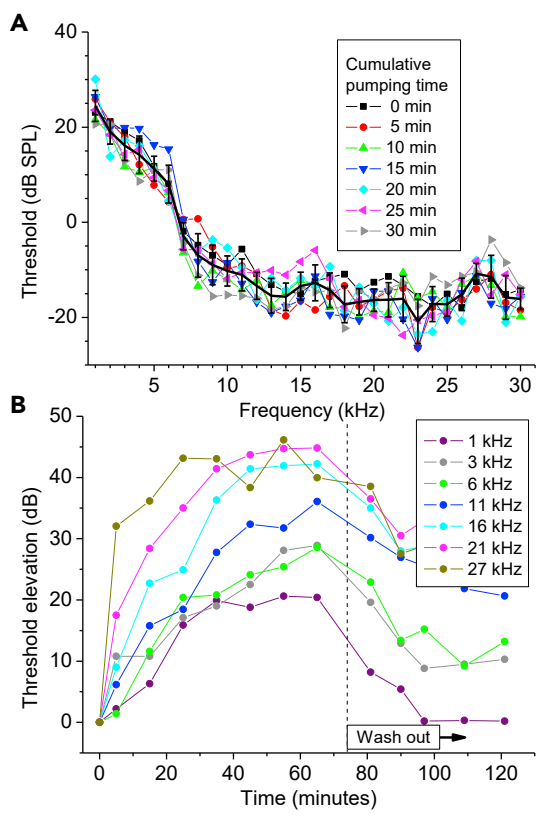

Figure 1. Effect of the CP on the Neural Responses in the Absence and Presence of Salicylate on the RW

(A) Representative examples of CAP threshold curves in the presence of $\mathrm{CP}$ alone. Pressure oscillations at $4 \mathrm{~Hz}$ that caused $\sim 80 \mu \mathrm{m}$ peak-to-peak stapes displacement were applied to the ear canal during 5 min before each non-zero time point plotted, and the CAP thresholds were measure during the following 5-min interval without $\mathrm{CP}$. The legend indicates the cumulative pumping time for each curve. Solid black line and vertical bars indicate the mean \pm SD for all seven curves, each representing a different cumulative pumping time.

(B) A representative example of CAP threshold elevation when the same pumping protocol as in (A) was used after the application of $5 \mu \mathrm{L}$ of $100 \mathrm{mM}$ salicylate solution to the RW at time zero and recovery of the thresholds after washing out the salicylate. The frequency of the pure tone acoustic stimulation used for eliciting the CAP is indicated in the figure legend for each curve.

from the RW (Figure 1B) provided confirmation that the integrity of the sensory cells was preserved and the threshold elevation after joint salicylate application and CP was not caused by the low-frequency pressure oscillations (Figure 1A).

The technique had no observable influence, compared with passive diffusion, on responses to RW salicylate application for locations close to the RW at the base of the cochlea (Figure 2, $27 \mathrm{kHz}$ ). CP, however, led to more rapid threshold elevation for locations that were distal and apical to the RW, even if the maximal threshold elevations were similar for both experimental paradigms (Figure 2, $15 \mathrm{kHz}$ ). The threshold elevation saturated after $4-5$ cycles of $C P$, even for the low frequencies of the most apical locations (Figures 1B and 2, 1-3 kHz) where passive diffusion produced no effect. Smaller threshold elevations at low frequencies were due only to the reduced contribution of cochlear amplification to cochlear responses at these frequencies (Sadreev et al., 2019; Robles and Ruggero, 2001) and, in fact, reached almost maximal possible elevations for those frequencies (Figure 3).

\section{DISCUSSION}

A stapes displacement of $80 \mu \mathrm{m}$ in our experiments corresponds to $1.6 \mathrm{~mm}$ linear displacement of the fluid in the apical parts of ST, because in guinea pigs the apical ST cross-sectional area is almost 20 times smaller (Thorne et al., 1999) than the stapes area (Sim et al., 2013) and both the cochlear bony wall and fluid are poorly compressible. Therefore, although salicylate effect in the most apical $25 \%$ of the cochlear length was not measured due to poor hearing sensitivity of guinea pigs below $1 \mathrm{kHz}$, most of the fluid in this region was replaced by fluid from more basal regions during a single cycle of $\mathrm{CP}$. Hence, estimates of salicylate distribution derived for the basal regions are valid for the most apical $25 \%$ of the cochlea.

The cochlear bony wall and fluid are poorly compressible. The poor compressibility results in the fluid volume velocity along the SV and ST being the same. Consequently, fluid linear displacement and velocity are much higher in the narrow apical parts of the scalae than at the base. Specifically, the apical ST crosssectional area in guinea pigs is almost 20 times smaller than in the basal cochlear region (it is almost six times smaller in humans) (Thorne et al., 1999). This proportional increase in fluid displacement and velocity cannot alone produce effective drug mixing along the cochlea. It will, however, facilitate mechanisms discussed below, which can enhance the distribution to the cochlear apex of a drug that originally diffuses through the RW and oval window into the cochlear base.

The larger fluid linear velocity at the apex is still not sufficient to cause turbulent mixing of drugs. Owing to the small diameter of the cochlear scalae, the fluid flow along them is dominated by fluid viscosity (i.e., it 

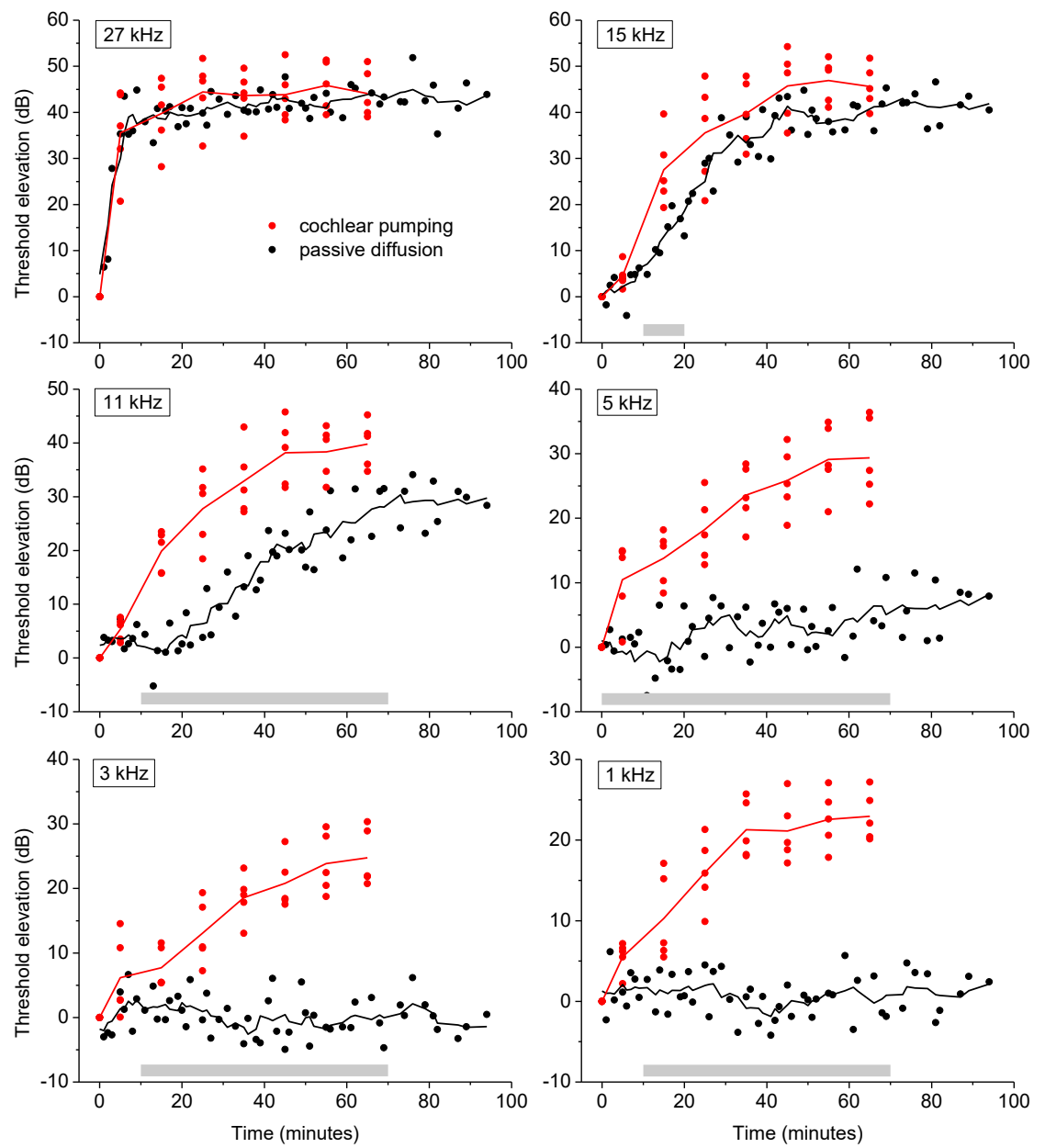

Figure 2. Comparison of the Efficiency of the CP Technique and Passive Diffusion in the Distribution of Salicylate along the Cochlea

Pooled data for experiments with CP (red symbols, five preparations) and passive diffusion (black symbols, five preparations; Sadreev et al., 2019). Pressure oscillations at $4 \mathrm{~Hz}$ were applied to the ear canal during 5 min before each red non-zero time point plotted to cause large-amplitude ( $80 \mu \mathrm{m}$ peak-to-peak) stapes movement, and the CAP thresholds were measured during the following 5 -min interval without pressure oscillation. Frequency of acoustic stimulation is indicated within each panel; $5 \mu \mathrm{L}$ of $100 \mathrm{mM}$ salicylate solution was applied to the RW at time zero. Solid red lines indicate mean CP data, and solid black lines indicate 5-point running averages for passive diffusion data. Gray lines near the horizontal axis indicate statistically significant $(p<0.05$, unpaired t test) differences between data for the CP and passive diffusion within consecutive 10-min intervals. Some of the passive diffusion data have been presented before (Sadreev et al., 2019).

occurs at low Reynolds numbers). Thus, the fluid flow is laminar even over the uneven inner surface (Cervo et al., 2013) of the scalae and turbulent mixing will not contribute to uniform drug distribution. The boundary layer-driven acoustic streaming (i.e., Rayleigh streaming) can potentially contribute to drug mixing in our experiments (Boluriaan and Morris, 2003; Squires and Quake, 2005). Its role, however, should not be significant because the boundary layer thickness in water/perilymph at $4 \mathrm{~Hz}$ is comparable with the ST diameter. Nevertheless, the cochlear helical structure should lead to additional drug mixing due to the formation of Dean vortexes (e.g., Nivedita et al., 2017) and to chaotic mixing/advection, both transversal and longitudinal, observed for laminar fluid flows in helical pipes (Jones et al., 1989; Nguyen, 2011), which can be further facilitated by periodic changes of the flow direction (Ottino and Wiggins, 2004). Therefore, under specific condition of cochlear stimulation, these mechanisms may well be the major factors contributing to the mixing and distribution of drugs along the ST and SV. None of the tentative mechanisms discussed above, except for acoustic streaming, causes net flow of perilymph along the scalae. We argue, however, that enhanced drug diffusion along the cochlea in our experiments could be observed without net forward 


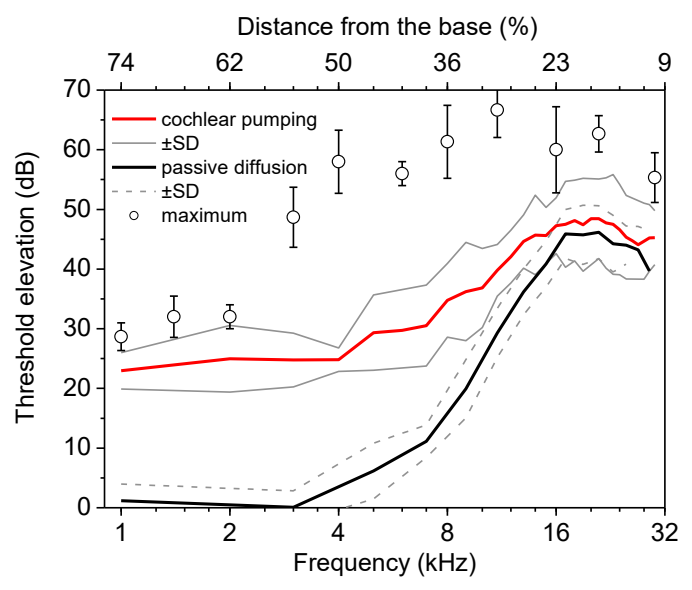

Figure 3. Comparison of Frequency Dependence of the $\mathrm{CP}$ and Passive Diffusion Effects

Frequency dependence of the CAP threshold elevation after $60 \mathrm{~min}$ of salicylate application during CP (35 min of the total pumping time) (mean $\pm S D, n=5$ ) and its comparison with the frequency dependence for passive diffusion (mean $\pm S D, n=5$ ). Open circles show maximal increase of the CAP thresholds after complete block of the cochlear amplifier (mean $\pm S D, n=3$ ) (Sadreev et al., 2019). Data for passive diffusion have been partially presented before (Sadreev et al., 2019).

motion of perilymph. Namely, we propose that the suggested mechanisms help to achieve more efficient mixing at the drug solution/pure perilymph borderline. Hence, this borderline moves much faster toward the apex than it is observed in experiments when only molecular diffusion is involved, which helps to achieve even drug distribution along the entire cochlea within a relatively short time.

The tentative physical principles that govern the uniform distribution of salicylate along the cochlea are universal and should be valid for the distribution of an arbitrary substance, including nanoparticles, in the human cochlea. Salicylate was used in these initial experiments because of its well-documented physiological effect, which allows estimation of the drug distribution along the intact cochlea without sampling perilymph. It was also used because it challenged the CP method. Salicylate is a difficult drug to distribute along the cochlea because it is cleared rapidly from the ST (Sadreev et al., 2019). It is anticipated that drugs, which are better retained in the ST, will be redistributed along the cochlea even more quickly and efficiently (Salt and Ma, 2001; Sadreev et al., 2019).

\section{Limitations of the Study}

Although the human cochlea has the same helical structure as the guinea pig cochlea, which should promote drug mixing due to the mechanisms discussed in the article, there are differences in the geometry of the cochlea that need to be considered for application of the CP in human patients. The human cochlea is longer, and the apical decrease in the cross-section of scalae is smaller than in guinea pigs (Thorne et al., 1999). This may result in longer time required to deliver drugs along the more extensive cochlea where increase in the liner fluid velocity at the apical part is less pronounced. On the other hand, the higher cutoff frequency and steeper low-frequency slope of the helicotrema's filter in humans (Marquardt et al., 2007) would permit the use of higher CP frequencies without damaging the cochlear sensory apparatus. This, together, with the possibility of using larger stapes displacements, which are limited to $\sim 80 \mu \mathrm{m}$ in guinea pigs by the crista stapedius, could result in higher CP efficiency.

Although prolonged, high-amplitude, low-frequency stimulation did not cause elevations of hearing thresholds (Figure 1A), this stimulation might potentially affect the vestibular system, especially the saccule. In fact, excitation of the saccule enables mice to detect low-frequency sounds, which are outside the frequency range of their cochleae (Jones et al., 2010). This possibility, however, could not be assessed under the neurolept anesthetic technique used in the study. Therefore, a thorough safety study of the CP method is needed for its translation to human patients.

\section{METHODS}

All methods can be found in the accompanying Transparent Methods supplemental file.

\section{SUPPLEMENTAL INFORMATION}

Supplemental Information can be found online at https://doi.org/10.1016/j.isci.2020.100945. 


\section{ACKNOWLEDGMENTS}

The research was funded by a grant from the Medical Research Council, United Kingdom (MR/N004299/1).

\section{AUTHOR CONTRIBUTIONS}

A.N.L., N.Z., Y.M.Y., and I.J.R. conceived and designed the study. A.N.L. performed the experiments. A.N.L. and I.I.S. analyzed experimental results. All authors contributed to analysis and discussion of the results. A.N.L. and I.J.R. wrote the manuscript with contribution from all authors.

\section{DECLARATION OF INTERESTS}

A.N.L., N.Z., and Y.M.Y. are inventors on a United Kingdom Patent Application No. 1908260.1 submitted by The University of Brighton that covers method and device for substance delivery to the inner ear. N.Z. is employed by the company Otophysica Ltd, Uckfield, UK. The remaining authors declare that the research was conducted in the absence of any commercial or financial relationships that could be construed as a potential conflict of interest.

Received: December 19, 2019

Revised: February 11, 2020

Accepted: February 21, 2020

Published: March 27, 2020

\section{REFERENCES}

von Békésy, G. (1960). Experiments in Hearing (McGraw-Hill).

Boluriaan, S., and Morris, P.J. (2003). Acoustic streaming: from Rayleigh to today. Int. J. Aeroacoust. 2, 255-292.

Borkholder, D.A., Zhu, X., Hyatt, B.T., Archilla, A.S., Livingston, W.J., III, and Frisina, R.D. (2010). Murine intracochlear drug delivery: reducing concentration gradients within the cochlea. Hear. Res. 268, 2-11.

Borkholder, D.A., Zhu, X., and Frisina, R.D. (2014). Round window membrane intracochlear drug delivery enhanced by induced advection.

J. Control. Release 174, 171-176.

Buckiová, D., Ranjan, S., Newman, T.A., Johnston, A.H., Sood, R., Kinnunen, P.K., Popelářr, J., Chumak, T., and Syka, J. (2012). Minimally invasive drug delivery to the cochlea through application of nanoparticles to the round window membrane. Nanomedicine 7, 1339-1354.

Cervo, DG Mansur, S. S, and Vieira, EDR. (2013). Flow over rough surfaces. In Proceedings of the 22nd International Congress of Mechanical Engineering (COBEM 2013), 3-7 November, 2013 (RibeirãoPreto Brazil), pp. 5966-5976.

Chen Z Kujawa, S.G. McKenna, M.J. Fiering J.O., Mescher, M.J., Borenstein, J.T., Swan, E.E.L., and Sewell, W.F. (2005). Inner ear drug delivery via a reciprocating perfusion system in the Guinea pig. J. Control. Release 110, 1-19.

Creber, N.J., Eastwood, H.T. Hampson, A.J. Tan, J., and O'Leary, S.J. (2018). A comparison of cochlear distribution and glucocorticoid receptor activation in local and systemic dexamethasone drug delivery regimes. Hear. Res. 368, 75-85.

Devare, J. Gubbels, S., and Raphael, Y. (2018) Outlook and future of inner ear therapy. Hear. Res. 368, 127-135.
Glueckert, R., Chacko, L.J., Rask-Andersen, H., Wei, L., Handschuh, S., and Schrott-Fischer, A (2018). Anatomical basis of drug delivery to the inner ear. Hear. Res. 368, 10-27.

Greenwood, D.D. (1990). A cochlear frequencyposition function for several species-29 years later. J. Acoust. Soc. Am. 87, 2592-2605.

Grewal, A.S., Nedzelski, J.M., Chen, J.M., and Lin V.Y. (2013). Dexamethasone uptake in the murine organ of Corti with transtympanic versus systemic administration. J. Otolaryngol. Head Neck Surg. 42,19

Haghpanahi, M., Gladstone, M.B., Zhu, X., Frisina R.D., and Borkholder, D.A. (2013). Noninvasive technique for monitoring drug transport through the murine cochlea using micro-computed tomography. Ann. Biomed. Eng. 41, 2130-2142.

Hallworth, R. (1997). Modulation of outer hair cell compliance and force by agents that affect hearing. Hear. Res. 114, 204-212.

Hao, J., and Li, S.K. (2019). Inner ear drug delivery: recent advances, challenges, and perspective. Eur. J. Pharm. Sci. 126, 82-92.

Hargunani, C.A., Kempton, J.B., DeGagne, J.M. and Trune, D.R. (2006). Intratympanic injection of dexamethasone: time course of inner ear distribution and conversion to its active form. Otol. Neurotol. 27, 564-569.

Imamura, S.I., and Adams, J.C. (2003)

Distribution of gentamicin in the Guinea pig inne ear after local or systemic application. J. Assoc. Res. Otolaryngol. 4, 176-195.

Jones, S.W., Thomas, O.M., and Aref, H. (1989). Chaotic advection by laminar flow in a twisted pipe. J. Fluid Mech. 209, 335-357.

Jones, G.P., Lukashkina, V.A., Russell, I.J., and Lukashkin, A.N. (2010). The vestibular system mediates sensation of low-frequency sounds in mice. J. Assoc. Res. Otolaryngol. 11, 725-732.

El Kechai, N., Agnely, F., Mamelle, E., Nguyen, Y., Ferrary, E., and Bochot, A. (2015). Recent advances in local drug delivery to the inner ear. Int. J. Pharm. 494, 83-101.

Marquardt, T. Hensel, J., Mrowinski, D., and Scholz, G. (2007). Low-frequency characteristics of human and Guinea pig cochleae. J. Acoust. Soc. Am. 121, 3628-3638.

Mynatt, R., Hale, S.A., Gill, R.M., Plontke, S.K., and Salt, A.N. (2006). Demonstration of a longitudinal concentration gradient along scala tympani by sequential sampling of perilymph from the cochlear apex. J. Assoc. Res. Otolaryngol. 7, 182-193.

Nguyen, N.-T. (2011). Micromixers: Fundamentals, Design and Fabrication (William Andrew).

Nivedita, N., Ligrani, P., and Papautsky, I. (2017). Dean flow dynamics in low-aspect ratio spiral microchannels. Sci. Rep. 7, 44072.

Ohyama, K., Salt, A.N., and Thalmann, R. (1988). Volume flow rate of perilymph in the Guinea-pig cochlea. Hear. Res. 35, 119-129.

Ottino, J.M., and Wiggins, S.R. (2004) Introduction: mixing in microfluidics. Philos. Trans. A Math. Phys. Eng. Sci. 362, 923-935.

Plontke, S.K., Mynatt, R., Gill, R.M., Borgmann, S., and Salt, A.N. (2007). Concentration gradient along the scala tympani after local application of gentamicin to the round window membrane. Laryngoscope 117, 1191-1198.

Plontke, S.K., Biegner, T., Kammerer, B., Delabar, U., and Salt, A.N. (2008). Dexamethasone concentration gradients along scala tympani after application to the round window membrane. Otol. Neurotol. 29, 401-406 
Ramaswamy, B., Roy, S., Apolo, A.B., Shapiro, B., and Depireux, D.A. (2017). Magnetic nanoparticle mediated steroid delivery mitigates cisplatin induced hearing loss. Front. Cell. Neurosci. 11 268

Rivera, T., Sanz, L., Camarero, G., and VarelaNieto, I. (2012). Drug delivery to the inner ear: strategies and their therapeutic implications for sensorineural hearing loss. Curr. Drug Deliv. 9, 231-242.

Robles, L., and Ruggero, M.A. (2001). Mechanics of the mammalian cochlea. Physiol. Rev. 81, 1305-1352.

Russell, I.J., and Schauz, C. (1995). Salicylate ototoxicity: effects on stiffness and electromotility of outer hair cells isolated from the Guinea pig cochlea. Auditory Neurosci. 1, 309-319.

Sadreev, I.I., Burwood, G.W.S., Flaherty, S.M., Kim, J., Russell, I.J., Abdullin, T.I., and Lukashkin, A.N. (2019). Drug diffusion along an intact mammalian cochlea. Front. Cell. Neurosci. 13, 161.

Saijo, S., and Kimura, R.S. (1984). Distribution of HRP in the inner ear after injection into the middle ear cavity. Acta Otolaryngol. 97, 593-610.

Salt, A.N., and Hirose, K. (2018). Communication pathways to and from the inner ear and their contributions to drug delivery. Hear. Res. 362, 25-37.

Salt, A.N., and Ma, Y. (2001). Quantification of solute entry into cochlear perilymph through the round window membrane. Hear. Res. 154, 88-97.

Salt, A.N., and Plontke, S.K. (2009). Principles of local drug delivery to the inner ear. Audiol. Neurotol. 14, 350-360.

Schilder, A.G.M., Su, M.P., Blackshaw, H., Lustig L., Staecker, H., Lenarz, T., Safieddine, S., GomesSantos, C.S., Holme, R., and Warnecke, A. (2019) Hearing protection, restoration, and regeneration: an overview of emerging therapeutics for inner ear and central hearing disorders. Otol. Neurotol. 40, 559-570.

Schuknecht, H.F. (1956). Ablation therapy for the relief of Meniere's disease. Laryngoscope 66, 859.

Sim, J.H., Röösli, C., Chatzimichalis, M., Eiber, A., and Huber, A.M. (2013). Characterization of stapes anatomy: investigation of human and Guinea pig. J. Assoc. Res. Otolaryngol. 14, 159-173.

Squires, T.M., and Quake, S.R. (2005).

Microfluidics: fluid physics at the nanoliter scale. Rev. Mod. Phys. 77, 977

Thorne, M., Salt, A.N., DeMott, J.E., Henson, M.M., Henson, O.W., Jr., and Gewalt, S.L. (1999) Cochlear fluid space dimensions for six species derived from reconstructions of three dimensional magnetic resonance images. Laryngoscope 109, 1661-1668. 
iScience, Volume 23

Supplemental Information

\section{Local Drug Delivery to the Entire Cochlea}

without Breaching Its Boundaries

Andrei N. Lukashkin, Ildar I. Sadreev, Natalia Zakharova, Ian J. Russell, and Yury M. Yarin 


\section{Transparent Methods}

Animals

Animal preparation and signal generation and recording have been described elsewhere (Burwood et al., 2017). Briefly, pigmented guinea pigs of similar weight (350-360 g) and both sexes were anaesthetised with the neurolept anaesthetic technique $(0.06 \mathrm{mg} / \mathrm{kg}$ body weight atropine sulphate s.c., $30 \mathrm{mg} / \mathrm{kg}$ pentobarbitone i.p., $500 \mu \mathrm{l} / \mathrm{kg}$ Hypnorm i.m.). Additional injections of Hypnorm were given every 40 minutes. Additional doses of pentobarbitone were administered as needed to maintain a non-reflexive state. The heart rate was monitored with a pair of skin electrodes placed on both sides of the thorax. The animals were tracheotomized and artificially respired with a mixture of $\mathrm{O}_{2} / \mathrm{CO}_{2}$, and their core temperature was maintained at $38^{\circ} \mathrm{C}$ with a heating blanket and a heated head holder.

All procedures involving animals were performed in accordance with UK Home Office regulations with approval from the University of Brighton Animal Welfare and Ethical Review Body.

\section{Signal generation and recording}

The middle ear cavity of the ear used for the measurements and salicylate application was opened to reveal the RW. Compound action potentials (CAPs) of the auditory nerve in response to pure tone stimulation were measured from the cochlear bony ridge in the proximity of the RW membrane using Teflon-coated silver wire coupled to laboratory designed and built extracellular amplifier (James Hartley). Thresholds of the N1 peak of the CAP at different frequencies which corresponds to different distances from the cochlear base (Greenwood, 1990) were estimated visually using $10 \mathrm{~ms}$ pure tone stimuli at a repetition rate of $10 \mathrm{~Hz}$.

For acoustic stimulation sound was delivered to the tympanic membrane by a closed acoustic system comprising two Bruel and Kjaer 4134 1/2" microphones for delivering tones and a single Bruel and Kjaer 4133 1/2" microphone for monitoring sound pressure at the tympanum. The microphones were coupled to the ear canal via $1 \mathrm{~cm} l o n g, 4 \mathrm{~mm}$ diameter tubes to a conical speculum, the $1 \mathrm{~mm}$ diameter opening of which was placed about $1 \mathrm{~mm}$ from the tympanum. The speculum was sealed in the ear canal. The closed sound system was calibrated in situ for frequencies between 1 and $50 \mathrm{kHz}$. Known sound pressure levels were expressed in dB SPL re $2 \times 10^{-5} \mathrm{~Pa}$.

All acoustic stimuli in this work were shaped with raised cosines of $0.5 \mathrm{~ms}$ duration at the beginning and at the end of stimulation. White noise for acoustical calibration and tone sequences for auditory stimulation were synthesised by a Data Translation 3010 board (Measurement Computing Corporation, MA) at $250 \mathrm{kHz}$ and delivered to the microphones through low-pass filters (100 kHz cut-off frequency). Signals from the acoustic measuring amplifier (James Hartley) were digitised at $250 \mathrm{kHz}$ using the same board and averaged in the time domain. Experimental control, data acquisition and data analysis were performed using a PC with programmes written in MATLAB (MathWorks, MA).

\section{Salicylate application}

$5 \mu \mathrm{l}$ of $100 \mathrm{mM}$ sodium salicylate solution in Hanks' Balanced Salt Solution were placed on the RW using pipettes. The solution was removed from the RW using paper wicks to observe the wash out effect.

\section{Generation of pressure oscillations in the ear canal}

A modified mouse ventilator MiniVent Type 845 (Hugo Sachs Elektronik, March, Germany) was used to generate oscillating air pressure in the ear canal. Output of the ventilator was connected and sealed to the closed acoustic system. The stroke frequency $(4 \mathrm{~Hz})$ and stroke volume $(150 \mu \mathrm{l})$ were the same for the all experiments reported. The stroke volume was maximized to achieve maximum stapes displacement limited only by the crista stapedius.

\section{Recording of stapes vibrations}

Stapes vibrations were recorded using a CLV-2534 laser vibrometer (Polytec GmbH, Waldbronn, Germany). The laser beam was focussed onto the stapes head. The output voltage from the vibrometer was low-pass filtered at 100 $\mathrm{kHz}$, with a sensitivity of $5 \mathrm{~mm} / \mathrm{s} / \mathrm{V}$. Stapes displacement was found by integrating the velocity responses off-line.

\section{Supplemental References}

Burwood, G.W.S., Russell, I,J., and Lukashkin, A.N. (2017). Rippling pattern of distortion product otoacoustic emissions evoked by high-frequency primaries in guinea pigs. J. Acoust. Soc. Am. $142,855-862$. doi:10.1121/1.4998584 